N. N. Vakhania

Nagoya Math. J.

Vol. 57 (1975), 59-63

\title{
THE TOPOLOGICAL SUPPORT OF GAUSSIAN MEASURE IN BANACH SPACE
}

\author{
N. N. VAKHANIA
}

\section{Introduction.}

The main result of the present paper is the theorem 1 , which describes the topological support of an arbitrary Gaussian measure in a separable Banach space. This theorem will be proved after some discussion of the notion of support itself. But we begin with the reminder of the notion of covariance operator of a probability measure. This notion has a great importance not only for the description of support of Gaussian measures but also for the study of other problems in the theory of probability measures in linear spaces (c.f. [1], [2]).

Let $X$ be a real Banach space with topological dual $X^{*}$ and $\mu$ be a probability measure on the $\sigma$-algebra generated by $X^{*}$. We suppose that $\mu$ has the second order in weak sense, i.e. $X^{*} \subset L_{2}(X, \mu)$ (this restriction is necessary if we want to speak about an analogue of variance). Covariance operator $R$ of measure $\mu$ is defined by the relation (see [1])

$$
\left(R x_{2}^{*}\right)\left(x_{1}^{*}\right)=\int_{X} x_{1}^{*}(x) x_{2}^{*}(x) \mu(d x)-\int_{X} x_{1}^{*}(x) \mu(d x) \int_{X} x_{2}^{*}(x) \mu(d x) .
$$

Here $\left(R x_{2}^{*}\right)\left(x_{1}^{*}\right)$ is the value of the linear functional $R x_{2}^{*} \in X^{* *}$ on the element $x_{1}^{*} \in X^{*}$. The operator $R$ defined by this relation is a linear, bounded, symmetrical $\left(\left(R x_{2}^{*}\right)\left(x_{1}^{*}\right)=\left(R x_{1}^{*}\right)\left(x_{2}^{*}\right)\right)$ and non-negative $\left(\left(R x^{*}\right)\right.$ $\cdot\left(x^{*}\right) \geqslant 0$ for all $\left.x^{*} \in X^{*}\right)$ mapping of $X^{*}$ into $X^{* *}$.

\section{On topological support of a probability measure.}

The support $S_{\mu}$ of a probability measure $\mu$ in a Banach space is by definition the smallest closed (measurable) set having $\mu$-measure 1. There exists another definition: the support $S_{\mu}^{\prime}$ is the union of all those points of the space, every measurable neighborhood of which has positive $\mu$ measure. It is obvious that $S_{\mu}^{\prime}$ always exists (the case of empty set is

Received July 15, 1974. 
not excluded) and besides if $S_{\mu}$ exists then $\mu\left(S_{\mu}\right)=1$. It is not difficult to show that if one of the following assertions:

(a) $S_{\mu}$ exists

(b) $\mu\left(S_{\mu}^{\prime}\right)=1$

is true then the another is also true and $S_{\mu}=S_{\mu}^{\prime}$. Indeed let $S_{\mu}$ exist and $O_{x}$ be a neighborhood of $x \in S_{\mu}$. If $\mu\left(O_{x}\right)=0$ then the closed set $S_{\mu} \backslash O_{x}$ must have measure 1. But it is impossible because $S_{\mu}$ is the smallest closed set of measure 1 . Consequently, $S_{\mu} \subset S_{\mu}^{\prime}$. If $x \bar{\in} S_{\mu}$ and $x \in S_{\mu}^{\prime}$ then there exists a neighborhood $O_{x}$ such that $O_{x} \cap S_{\mu}$ is empty and $\mu\left(O_{x}\right)>0$ (for example $\left.O_{x}=X \backslash S_{\mu}\right)$. But this is impossible as $\mu\left(S_{\mu}\right)=1$. Consequently $S_{\mu}=S_{\mu}^{\prime}$. Let now (b) be valid, i.e. $\mu\left(S_{\mu}^{\prime}\right)=1$. It is easy to see that $S_{\mu}^{\prime}$ is a closed set. Let us show that $S_{\mu}^{\prime}$ is the smallest (measurable) closed set of measure 1 . Let $S$ be closed, $\mu(S)=1$ and let $S_{\mu}^{\prime} \not \subset S$. Then there exists a neighborhood $O_{x}$ of some point $x \in S_{\mu}^{\prime}$ such that $O_{x} \cap S$ is empty and $\mu\left(O_{x}\right)>0$ (for example $O_{x}=X \backslash S$ ). But this contradicts to the supposition $\mu(S)=1$.

In the general (non-separable) case it may be that neither assertion (a) nor (b) is true. In [3] (see also [1]) for any given positive number $\rho$ the measure $\mu$ (Gaussian) in Banach space $\ell_{\infty}$ (on $\sigma$-algebra generated by $\left.\ell_{1} \subset \ell_{\infty}^{*}\right)$ is constructed such that $\mu$-measure of any ball of radius $\rho$ is zero. It is clear that for such measure the set $S_{\mu}^{\prime}$ is empty, i.e. the assertion (b) does not hold and therefore the assertion (a) is not true, i.e. the smallest closed (measurable) set having measure 1 does not exist. Consequently the definitions of support for a general case are out of use since the support in the first sense may not exist and in the second sense it may be even an empty set. In the separable case however these difficulties do not arise because the assertion (a) is always true. Indeed in this case the set $S_{\mu}$ can be obtained as an intersection of all closed sets having measure 1 . This possibility is an immediate consequence of the following elementary

LEMMA. In a separable space the intersection of any system of closed sets of measure 1 is again a closed set of measure 1.

The proof of this lemma is quite easy and it is ommited here.

\section{Support of Gaussian measure.}

Everywhere below $X$ will be assumed to be separable. In separable 
$X \sigma$-algebra generated by $X^{*}$ is the same as $\sigma$-algebra of Borelian sets. The definitions of $S_{\mu}$ and $S_{\mu}^{\prime}$ are correct and $S_{\mu}=S_{\mu}^{\prime}$. Note that in the case of separable $X$ if $R: X^{*} \rightarrow, X^{* *}$ is the covariance operator of Gaussian measure then $R X^{*} \subset X$ in the sense of natural embedding of $X$ into $X^{* *}$ (see [1]). Thus taking into consideration that this natural embedding is a linear isomorphism we can simply mean that in a separable case the operator $R$ maps $X^{*}$ into $X$.

Let $\overline{R X}^{*}$ denote the closure in $X$ of the set $R X^{*}$. Furthermore let $\mathfrak{m}$ denote the mathematical expectation (mean value) of measure $\mu$ defined, as it is well known, in the sense of Pettis integral (i.e. $x^{*}(\mathfrak{m})=$ $\int_{X} x^{*}(x) \mu(d x)$ for all $\left.x^{*} \in X^{*}\right)$.

THEOREM 1. $S_{\mu}=\mathfrak{m}+\overline{R X}^{*}$ for any Gaussian measure $\mu$ in $X$ with expectation $\mathfrak{m}$ and covariance operator $R$.

Proof. If we transform a measure according to the translation of the space, the support will be also translated by the same element. Therefore it is enough to consider the case $\mathfrak{m}=0$. From the results given in H. Sato's paper [4] it follows immediately that $S_{\mu}$ is a linear manifold in case $\mathfrak{m}=0$ (recently Nguen zui Tien has given immediate and elementary proof of this fact). The linear subspace in $X$ is also $\overline{R X^{*}}$. Thus it is enough to show that

$$
\overline{R X}^{* \perp}=S_{\mu}^{\perp},
$$

where $A^{\perp}$ denotes as usual the annihilator of $A$ i.e. the set of those points $x^{*} \in X^{*}$ for which $x^{*}(x)=0$ everywhere in $A \subset X$. Now let us prove the equality (2).

(a) The proof of inclusion $\overline{R X^{*} \perp} \subset S_{\mu}^{\perp}$.

Let $y^{*} \in \overline{R X^{*} \perp}$. This assumption is equivalent to the equality $y^{*}\left(R x^{*}\right)$ $=0$ for all $x^{*} \in X^{*}$. In particular, remembering the definition (1) of covariance operator, we have

$$
y^{*}\left(R y^{*}\right)=\int_{X} y^{* 2}(x) \mu(d x)=\int_{S_{\mu}} y^{* 2}(x) \mu(d x)=0
$$

and consequently

$$
\mu\left\{x: y^{*}(x)=0\right\}=1
$$


But the set $\left\{x: y^{*}(x)=0\right\}$ is closed and has measure 1 , hence it contains $S_{\mu}$ i.e. $y^{*}(x)=0$ for all $x \in S_{\mu}$. This means that $y^{*} \in S_{\mu}^{\perp}$ what was to be proved.

(b) The proof of inclusion $S_{\mu}^{\perp} \subset \overline{R X^{*} \perp}$.

Let $y^{*} \in S_{\mu}^{\perp}$ i.e. $y^{*}(x)=0$ everywhere on $S_{\mu}$. Then denoting by $\chi(\cdot ; \mu)$ the characteristic functional of the measure $\mu$ we have

$$
\chi\left(y^{*} ; \mu\right)=\int_{X} \exp \left\{i y^{*}(x)\right\} \mu(d x)=\int_{S_{\mu}} \exp \left\{i y^{*}(x)\right\} \mu(d x)=1
$$

It is easy to see that the characteristic functional of the Gaussian measure $\mu$ with covariance operator $R$ and expectation zero has the form

$$
\chi\left(x^{*} ; \mu\right)=\exp \left\{-\frac{1}{2} x^{*}\left(R x^{*}\right)\right\}, \quad x^{*} \in X^{*},
$$

and thus from the equality (3) we obtain

$$
y^{*}\left(R y^{*}\right)=0 \text { for } y^{*} \in S_{\mu}^{\perp}
$$

Now we have to use the following elementary inequality the proof of which does not differ from the proof of the corresponding well known inequality in Hilbert space:

$$
\left|y^{*}\left(R x^{*}\right)\right|^{2} \leq y^{*}\left(R y^{*}\right) x^{*}\left(R x^{*}\right), \quad x^{*}, y^{*} \in X^{*}
$$

This inequality (which is valid for any linear bounded symmetrical non-negative mapping of $X^{*}$ into $X$ ) shows that it follows from (4) the condition: $y^{*}\left(R x^{*}\right)=0$ for all $x^{*} \in X^{*}$. But it means that $y^{*} \in \overline{R X}^{* \perp}$.

The proof is complete.

COROLLARY. In accordance with the definition in finite-dimensional space, a probability measure $\mu$ on a Banach space $X$ is called non-degenerate if every non-trivial linear functional $x^{*} \in X^{*}$ as a random variable on $(X, \mu)$ has non-degenerate (i.e. non-concentrated at a single point) distribution. Since the variance of $x^{*}$ is $\left(R x^{*}\right)\left(x^{*}\right)$ non-degeneracy means that $\left(R x^{*}\right)\left(x^{*}\right)=0$ only for $x^{*}=0$. Using the elementary inequality (5) and the Hahn-Banach theorem, it is easy to show in the same manner as it is done in [5] for the case $X=\ell_{p}$ that the following two assertions are equivalent and each of them also means non-degeneracy of $\mu$ :

(a) The mapping $R: X^{*} \rightarrow X$ is one to one,

(b) $\overline{R X^{*}}=X$. 
Let now $\mu$ be a Gaussian measure. According to Theorem 1 and the assertion (b) non-degeneracy of $\mu$ means that $S_{\mu}=X$ (essentially this fact is a consequence of the linearity of the support). Consequently for any Gaussian measure $\mu$ in separable $X \mu$-measure of every non-empty open set is positive (in non-separable space the matter is much more complicated, see [3]).

Remark. Theorem 1 contains B. Rajput's recent result ([6], the case $X=\ell_{p}, 1 \leq p<\infty$ ) and earlear result of $\mathrm{K}$. Ito ([7], the case of separable Hilbert space). The reasoning used to prove Theorem 1 are contained in our paper [5] which contains even the proof of Theorem 1 for the case $X=\ell_{p}$ assuming non-degeneracy of the measure. Note that the limitation of non-degeneracy is not essential and using a little additional argument we can come to the general case (see [8], where it is done for the case $p=2$ ).

\section{REFERENCES}

[ 1 ] Вахания, Н. Н., Вероятностные распределения в линейных пространствах. Тбилиси, 1971.

[2] Vakhania, N. N., On some questions of the theory of probability measures on Banach spaces. Lecture note at Nagoya University, 1973.

[ 3 ] Vakhania, N. N., On a property of Gaussian distributions in Banach spaces. Sankhya, The Journal of Indian Statistical Society. Series A, vol. 35 (1973), pp. 23-28.

[4] Sato, H., Gaussian measures on Banach space and abstract Wiener measure. Nagoya Math. J., vol. 36 (1969), pp. 65-81.

[ 5 ] Вахания, Н. Н., О невырожденных распределениях вероятностей в пространствах $\ell_{p}(1 \leq p<\infty)$. Теория вероятн. и ее применен., т. II, No. 3, (1966), стр. 524-528.

[6] Balram S. Rajput, The support of Gaussian measures on Banach spaces. Теория вероятн. и ее применен., т. 27, No. 4, (1972), стр. 775-782.

[ 7 ] Ito, K., The topological support of Gauss measure on Hilbert space. Nagoya Math. J., vol. 38 (1970), pp. 181-183.

[ 8 ] Vachania, N. N., Träger des Gausschen masses im Hilbertraum. Mathem. Nachrichten 64. Band (1974), pp. 319-322.

Tbilisi University, USSR 